\title{
A radiological study method of vertical alveolar resorptions using immediate dental implants
}

\author{
Dragos Epistatu ${ }^{1}$, Andreea Ciolacu', Aikaterina Kitsou', Ioanina Parlatescu ${ }^{2}$, Octavian Dinca $^{3}$, \\ Cristian Vladan ${ }^{3}$, Florin Dogioiu', Valentin Varlas ${ }^{4,5}$, Paula Perlea ${ }^{6}$ \\ ${ }^{1}$ Department of Dental Radiology, Faculty of Dental Medicine, \\ "Carol Davila" University of Medicine and Pharmacy, Bucharest, Romania \\ ${ }^{2}$ Department of Oral Pathology, Faculty of Dental Medicine, \\ "Carol Davila" University of Medicine and Pharmacy, Bucharest, Romania \\ ${ }^{3}$ Department of Oral and Maxillofacial Surgery, Faculty of Dental Medicine, \\ "Carol Davila" University of Medicine and Pharmacy, Bucharest, Romania \\ ${ }^{4}$ Department of Obstetrics and Gynecology, "Carol Davila" University of Medicine and Pharmacy, Bucharest, Romania \\ ${ }^{5}$ Department of Obstetrics and Gynecology, Filantropia Clinical Hospital, Bucharest, Romania \\ ${ }^{6}$ Department of Endodontics, Faculty of Dental Medicine, "Carol Davila" University of Medicine and Pharmacy, \\ Bucharest, Romania
}

\begin{abstract}
Dental extraction is followed by decreases in alveolar volume, vertically and horizontally. This retrospective study measured vertical alveolar resorption after insertion of immediate implants, using OPG, without CBCT or additional radiographs. Patients were randomly splitted in two groups A ( 57 implants) and B (47 implants). Measurements were made by two independent observers. The types of implants were: Exacta (Biaggini Medical Devices), Nova, Dentix Millenium and Surcam. The method was based on the size of the implant and another common element of the two radiographs. It used an optimal radiologic exposure. It can be generalized to other studies. Similar results measured in the two groups showed that alveolar remodelling does not depend on the type of the implant. Half of the alveoli did not have any resorption. Another quarter showed barely perceptible clinical resobtion (1-2 $\mathrm{mm})$. In the last quarter were registered all the notable resorptions that were associated with various factors (temporary mobile prosthesis, surgical flap, medication and some health problems, smoking, etc) showing real situations, not ideal conditions. Maximum resorption was unique $(7.63 \mathrm{~mm})$.
\end{abstract}

Keywords: immediate implants, vertical resorption, radiologic measurement method

\section{INTRODUCTION}

Dental extraction is followed by remarkable bone changes, including decreases in alveolar volume, vertically and horizontally. These may jeopardize the subsequent application of the implants. At 6 months after extraction, the resorption of the vestibular wall is $19.4 \pm 9.4 \%$ at midpoint, $39.1 \pm$ $10.4 \%$ at the central point and $20.3 \pm 10.7 \%$ at the distal point [1]. We can therefore witness a reduction of up to $50 \%$ in vertical direction of this wall.
The average buccolingual size can also decrease by approx. $30 \%$. A very credible study shows the extraction value of $10.5 \mathrm{~mm}( \pm 1.52)$ and the discovery of the prosthesis implant $6.8 \mathrm{~mm}( \pm 1.33)$, without guided tissue regeneration [2].

A systematic review of the 2,009 articles (1244 Medline and 106 Cochrane) on the study of post-extractive alveolar changes selected only 12 as eligible. The conclusion referred to the reduction by $3.87 \mathrm{~mm}$ in the buccolingual direction and by 1.53 
$\mathrm{mm}$ in the vertical direction of the alveolar dimensions in the first 6 months after extraction [3]. From the above it seems that there is not too much uniformity of study methods and the results vary from study to study.

Alveolar remodelling seems inevitable. There are high-risk patients (thin periodontium) that reach up to $7.5 \mathrm{~mm}$ vertically [4]. Careful examination of the cases can make a prediction of possible accentuated remodelling. It is known that wearing a mobile prosthesis produces greater resorption of the alveolar process [5]. The association between diabetes and mechanical forces applied to the alveolar process leads to a potentiation of resorptions [6]. Certain systemic factors may increase alveolar resorption: decreased calcium absorption, osteoporosis, hyperthyroidism, hyperparathyroidism, some drugs (corticosteroids, thyroxine). The age and sex of the patient also have an important role [7].

A review of 1171 articles published in 2009-2017 clearly states that the success rate of immediate implants is excellent and that it does not matter much whether the initial sites are infected or not. Infections have been understood as foci of periodontal or apical problems [8]. We believe that the problem of diffuse osteitis should be better nuanced.

It is known that the buccal alveolar resorptions are the highest in the flap technique without addition material, then in the flap technique with addition material. The flapless application technique has the best results on actual resorption [9].

The study of post-extraction alveolar resorptions and remodeling can be done using СBCT type tomography [4]. However, the dose received would be significant given that the exposure should be repeated at least two or more times.

The study models obtained by classical impressions have the disadvantage of some dimensional changes, therefore they are not the best choice for the study of progressive resorptions. However, there is also the CAD / CAM scanning research variant [10] of the alveoli, which can non-invasively follow the external changes but does not provide information about a possible implant or about the bone structure.

\section{PURPOSE}

The aim of this study is to measure alveolar vertical resorption after insertion of immediate implants, using OPG measurements.

\section{MATERIAL AND METHODS}

The clinical cases included in the study were taken at random from the database of the Dr. Dragoş Epistatu Clinic, and the collection of general information was made from the patient observation sheet from 2009-2017. Patients were also randomly divided into two groups A (57 implants) And B (47 implants), In each group there was a random distribution of ages, male / female ratio and types of implants. The measurements were made by two independent observers for a better control of the subjective factor.

The types of implants used were: Exacta (Biaggini Medical Devices), Nova, Dentix Millennium and Surcam.

Patients completed a questionnaire on general health, performed blood tests (screening). Patients who had a contraindication to surgery or implantation were excluded, but patients with various pathologies with a maximum moderate risk for the success of implants were accepted (certain cardiovascular diseases, controlled diabetes, etc.). Smoking patients were warned of the risks but not excluded. Two patients underwent gynecological examinations before the operation (being in evidence), thus excluding the possibility of being pregnant at the time of implant insertion.

The causes of tooth extraction were: complicated carious lesions, coronary fractures, failures of endodontic treatments, periodontal damage that does not involve radiologically visible diffuse peri-radicular osteitis or found intraoperatively.

Tooth extraction was performed under local anesthesia with or without flap depending on the situation. An atraumatic operation was performed to preserve the alveolus, especially the alveolar vestibular wall. The implants were inserted in an optimal three-dimensional position: $1.5 \mathrm{~mm}$ from the adjacent teeth in the mesio-distal direction, 2-3 $\mathrm{mm}$ below the enamel-cement junction, and in the vestibulo-oral direction at 1-3 $\mathrm{mm}$ from the vestibular wall. Granular bone, Osteobiol or Geistlich type, was applied to dehiscences over $2 \mathrm{~mm}$ alveolo-implantation. Collagen membrane from the same manufacturers was applied to all of them. After inserting the implants, the suture was made, closing with a flap or suture in an "X" position that would leave a minimal dehiscence protected by the membrane. Antibiotic treatment was instituted before surgery and continued for 5-7 days. The temporary prosthesis was made predominantly jointly (fixed on the limiting teeth) but not exclusively. There were situations in which the application of temporary mobile prosthesis could not be avoided. After 4 and 6 months respectively from the surgical work, time necessary for the healing process, the clinical situation is re-evaluated both intraorally and radiologically and the treatment with the prosthetic stages is continued, in view of the final prosthesis.

The degree of alveolar resorption was measured on radiographs during two stages: T0 representing the initial situation, before the insertion of the im- 
plants, and T1 being at least 4 and 6 months after surgery.

Several parameters were measured in the study. For the current analysis, the following are taken into account initial height, final height, initial maximum alveolo-implant space, final peri-implant bone density.

\section{Correction factor}

Radiographs do not always have a 1:1 size scale; These are due to the x-ray machine or even the patient's positioning, and dimensional errors may occur. To improve these errors as much as possible, we introduced a radiography correction factor that represents the ratio between the actual dimensions and the dimensions measured on radiographs (e.g. dental implant). Thus, we performed a ratio between the real length of the implant (obtained from the patient's file) and the length of the implant measured on the radiograph performed 4-6 months after the surgical implant insertion procedure (T1). We considered that vertically this correction factor is maintained for all structures.

Then we identified a common element (length of a tooth) that appears on the initial (T0) and final (T1) radiograph. Using this common element we also calculated the correction factor (CF) for the initial radiograph (from $\mathrm{T} 0$ ).

$$
\begin{gathered}
\mathrm{CF}(\mathrm{T} 1) \times \mathrm{DRX} 1=\mathrm{CF}(\mathrm{T} 0) \times \mathrm{DRX} 0=\text { actual length of } \\
\text { the common element }
\end{gathered}
$$

where DRX0 = dimension of the common element measured on the initial radiograph (T0) and DRX1 = dimension of the same element measured on the final radiograph $(\mathrm{T} 1)$, so $\mathrm{CF}(\mathrm{T} 0)=\mathrm{CF}(\mathrm{T} 1) \mathrm{x} \mathrm{DRX} 1 /$ DRX0.

Because our study aimed to explore the resorption of the alveolar process at post-extractive im- plants, we chose to measure the distance from the edge of the alveolar process to a vertical structure on all radiographs and to compare the results. Depending on the area where the implants were inserted, we chose as landmarks the following anatomical formations: the floor of the nasal fossa (for the anterior maxillary region), the floor of the maxillary sinus (lateral maxillary area), the basilar edge of the mandible (anterior mandibular area) and mandibular canal (for the mandibular lateral region).

On the initial radiograph, $\mathrm{T} 0$, we measured the distance in the vertical plane from the alveolar process to one of the structures mentioned above, we multiplied the value obtained by the correction factor and determined the initial height (IH).

$\mathrm{IH}=$ (measured height on initial X-ray) $\mathrm{x}$ CF(T0)

On the final radiograph (T1), we identified points $A$ and $B$ corresponding to the mesial and distal walls of the alveolus in the horizontal plane. We determined half the distance between the two alveolar walls, denoted by $\mathrm{C}$, which corresponds to the middle portion of the implant neck. We measured vertically the distance from point $\mathrm{C}$ to one of the anatomical structures mentioned above, depending on the evaluated area. The result obtained represents the final height. In order for the values to be adequate, we multiplied the result by the determined correction factor and obtained the final height (FH).

$$
\mathrm{FH}=(\text { measured height on final } \mathrm{X} \text {-ray) } \mathrm{x} \mathrm{CF}(\mathrm{T} 1)
$$

\section{Determination of the alveolo-implant space}

The alveolo-implant space (AIS) represents the distance between the alveolar walls and the implant, measured in the mesio-distal direction. After measuring the distance A0-B0 (fig. 1) on the initial radiograph, we decreased the diameter of the in-

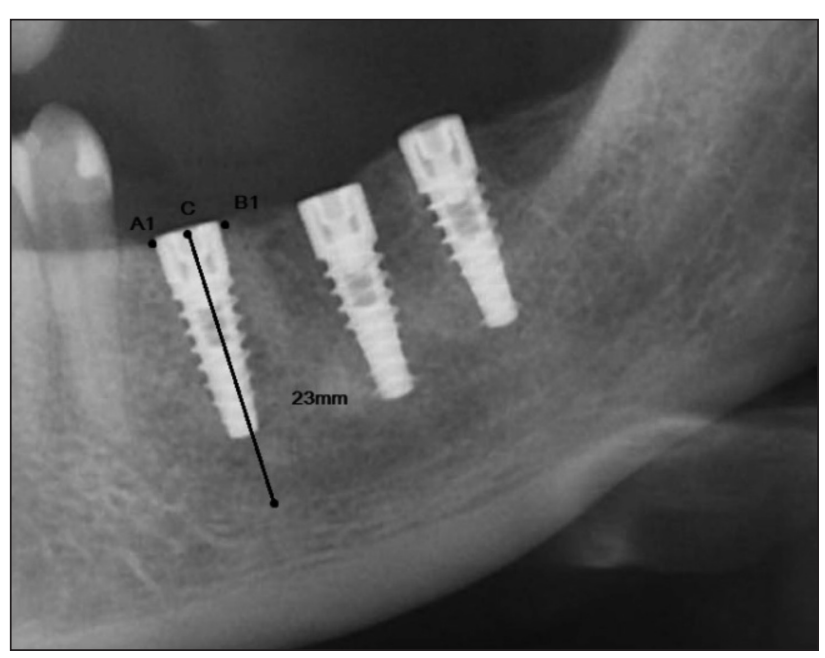

FIGURE 1. Determination of the initial and final heights in relation to the mandibular canal 
serted implant (known from the file) thus obtaining the initial value of the alveolo-implant space.

AIS $=$ [alveolar diameter on initial X-ray x FC(T0)] - (implant diameter)

\section{RESULTS}

The measured values vary between 0 and $6 \mathrm{~mm}$, a single clinical case having the maximum value. A number of 28 alveoli resulted in a perfect healing, the degree of resorption being non-existent, with a value of almost 0 . The rest showed some resorptions.

The values in both groups were then ordered in increasing intervals and graphs were made according to these values.

It is observed that half of the values are almost zero. Another quarter have a minimum value of

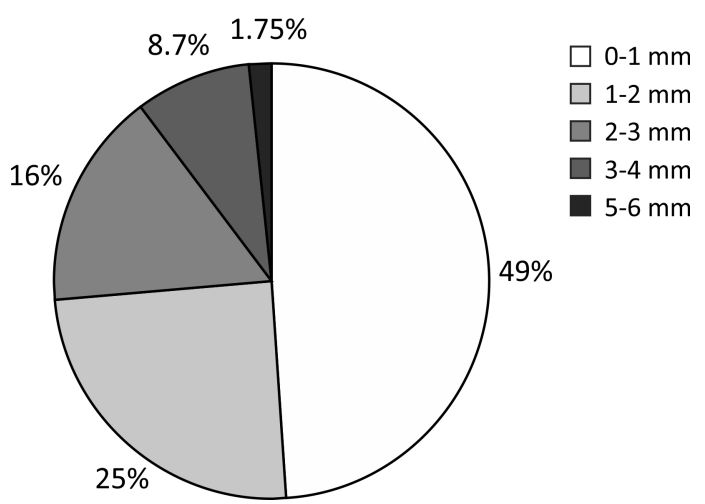

FIGURE 4. Percentage representation of value ranges in group A

1-2 mm. In the last quarter the increasing distribution of values varies in an inverse way with their percentage.

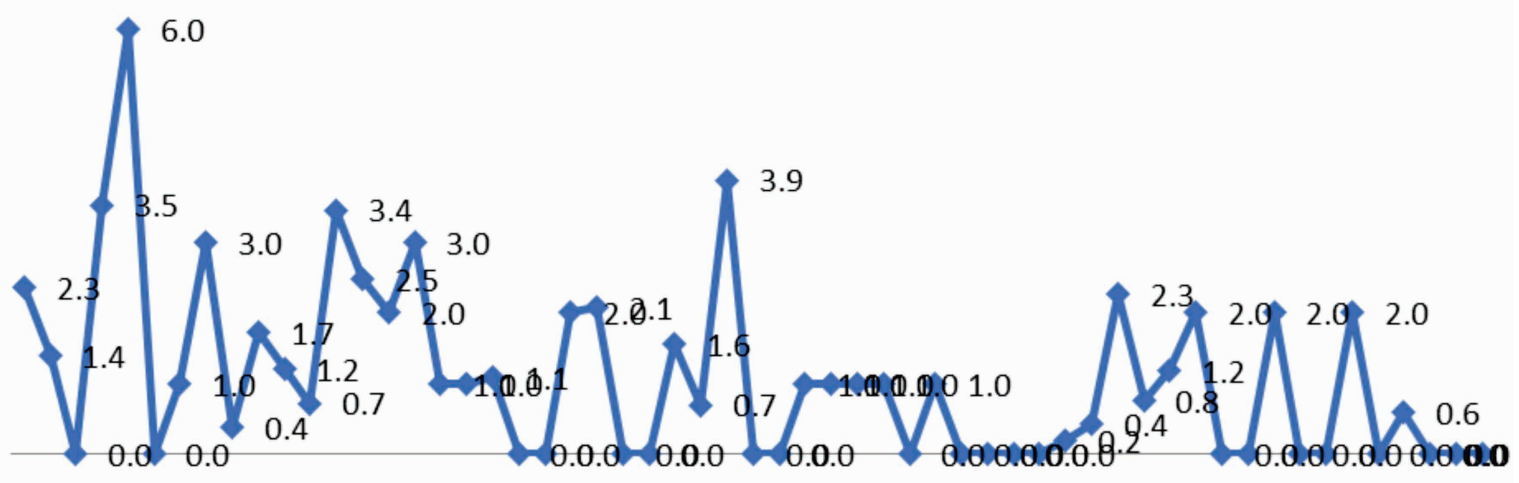

$\begin{array}{lllllllllllllllllllllllllllll}1 & 3 & 5 & 7 & 9 & 11 & 13 & 15 & 17 & 19 & 21 & 23 & 25 & 27 & 29 & 31 & 33 & 35 & 37 & 39 & 41 & 43 & 45 & 47 & 49 & 51 & 53 & 55 & 57\end{array}$

FIGURE 2. Values of vertical alveolar resorption at the 57 implants analysed in group $A$

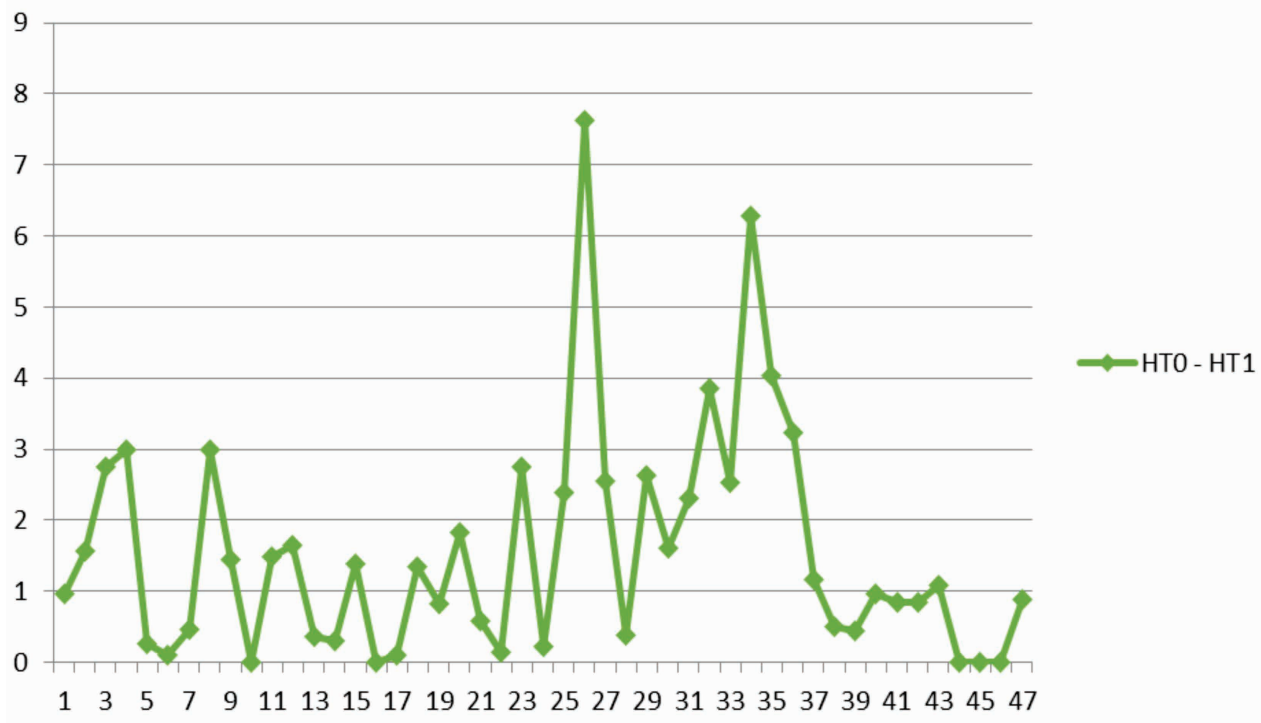

FIGURE 3. Alveolar resorption values for the 47 implants analysed in group B 


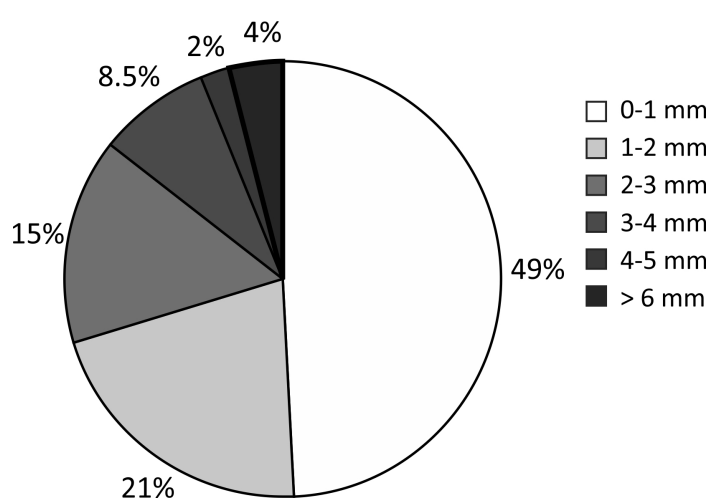

FIGURE 5. Percentage representation of value ranges in group $B$

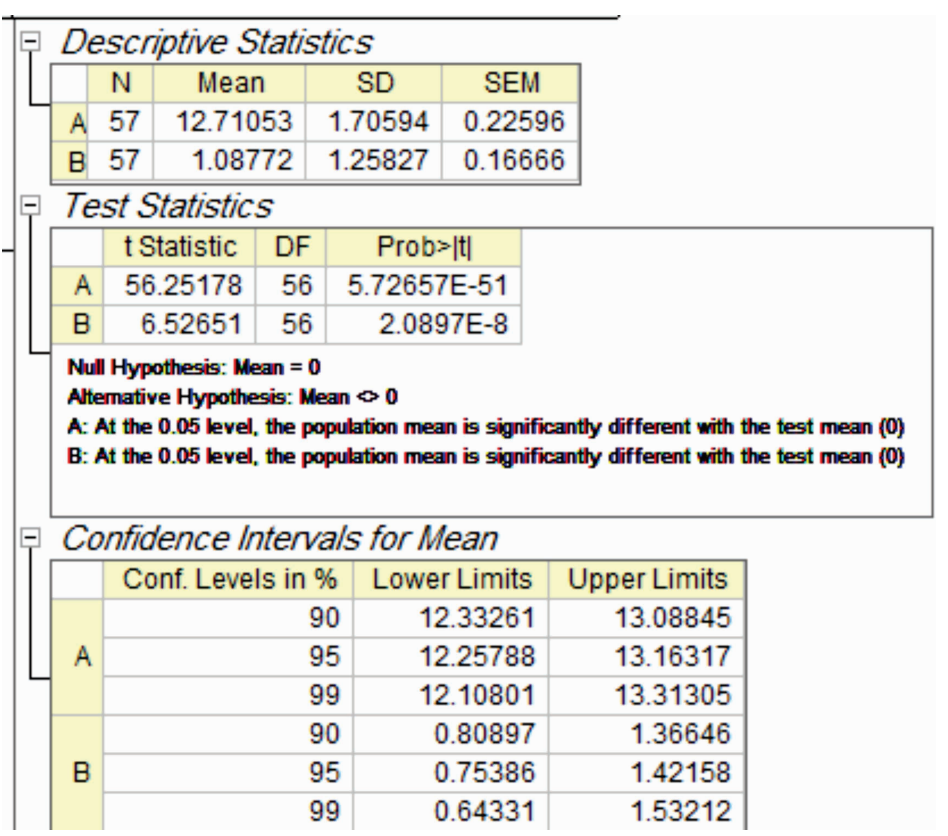

\section{\pm Input Data}

$\square$ Descriptive Statistics

\begin{tabular}{|c|c|c|c|c|c|c|c|c|c|c|c|c|}
\hline & N total & Mean & Standard Deviation & Sum & Minimum & Index of Minimum & Median & Maximum & Index of Maximum & P90 & P95 & P99 \\
\hline A & 57 & 12.71053 & 1.70594 & 724.5 & 10 & 21 & 13 & 18 & 23 & 15 & 15 & 18 \\
\hline B & 57 & 1.08772 & 1.25827 & 62 & 0 & 3 & 1 & 6 & 5 & 3 & 3.5 & 6 \\
\hline
\end{tabular}

\section{$\mp$ Input Data \\ $\mp$ Descriptive Statistics \\ NormalityTest

\begin{tabular}{|c|c|c|c|}
\hline \multicolumn{4}{|c|}{ Shapiro-Wilk } \\
\hline & DF & Statistic & Prob $<W$ \\
\hline A & 57 & 0.89774 & $1.60197 \mathrm{E}-4$ \\
\hline B & 57 & 0.82292 & $8.83638 \mathrm{E}-7$ \\
\hline
\end{tabular}

A: At the 0.05 level, the data was not significantly drawn from a normally distributed population B: At the 0.05 level, the data was not significantly drawn from a normally distributed population

\section{Kolmogorov Smirnov}

\begin{tabular}{|r|r|r|r|}
\hline & DF & Statistic & Prob>D \\
\hline A & 57 & 0.16947 & 0.067 \\
\hline B & 57 & 0.19367 & 0.02393 \\
\hline
\end{tabular}

A: At the 0.05 level, the data was significantly drawn from a normally distributed population B: At the 0.05 level, the data was not significantly drawn from a normally distributed population
It is observed that half of the values are close to zero. More than one-fifth have the minimum values. Almost a quarter had some bigger resorptions.

Regarding the alveolo-implant space (AIS), an attempt was made to correlate it statistically with vertical alveolar resorption. The results were not statistically significant, but a possible correlation with implant diameter is suggested, which remains to be further analysed in a later study.

\section{DISCUSSIONS}

The values obtained in the literature vary from one study to another, between zero and $7.5 \mathrm{~mm}$ in the vertical direction [4]. We found a range 0-7.63 $\mathrm{mm}$. It is observed that the two graphs obtained by 
us are very similar with the small exception of the field of higher resorptions. This confirms the normal distribution of cases in both groups, as they were chosen at random. The double analysis suggests the existence of a "pattern" of resorptions. Their causes are probably multiple (from flap technique, to osteo-gingival peculiarities, small associated health problems, medication etc.) $[4,6,7]$.

One thing that has been verified is that the higher resorptions were due to the wearing in the temporary stage of inevitable mobile prostheses that certainly shaped the alveolar healing [5]. However, high values were few.

OPG is the routine exploration method for implant placement. The radiograph shows globally other pathologies of the patient in the maxillofacial area. It is cheaper and exposes the patient to a dose several times lower than a CBCT [11]. The only possible further dose reduction would be the use of retro-alveolar radiographs in T1 because an OPG is always recommended in $\mathrm{T} 0$. (screening at the beginning of treatment). This only makes sense in the case of a single-tooth implant but is difficult in some patients with an exaggerated vomiting reflex or narrow arches.

A study of ridge height measured on OPG showed that there is a tendency to overestimate, due to the resorption of the vestibular portion that moves the tip of the ridge to the lingual [12]. This aspect is relative because in our study the measurement was made on OPG and in $\mathrm{T} 0$ and $\mathrm{T} 1$ and the placement of the implant immediately is not made in contact with the vestibular wall, as much as possible. Assuming this, we believe that the proposed method is good for retrospective analysis of activity in the dental office.

\section{REFERENCES}

1. Covani U, Ricci M, Bozzolo G, Mangano F, Zini A, Barone A. Analysis of the pattern of the alveolar ridge remodeling following single tooth extraction. Clin Oral Implants Res. 2011 Aug;22(8):820-5.

2. Covani U, Cornelini R, Barone A. Bucco-lingual bone remodeling around implants placed into immediate extraction sockets: a case series. J Periodontol. 2003 Feb;74(2):268-73.

3. Van der Weijden F, Dell'Acqua F, Slot DE. Alveolar bone dimensional changes of post-extraction sockets in humans: a systematic review. J Clin Periodontol. 2009 Dec;36(12):1048-58.

4. Chappuis V, Engel O, Reyes M, Shahim K, Nolte LP, Buser D. Ridge post-extraction alterations in the aesthetic zone: a $3 \mathrm{D}$ analysis with CBCT. J Dent Res. 2013 Dec;92(12 Suppl):195S-201S.

5. Ozan O, Orhan K, Aksoy S, Icen M, Bilecenoglu B, Sakul BU. The effect of removable partial dentures on alveolar bone resorption: a retrospective study with cone-beam computed tomography. J Prosthodont. 2013 Jan;22(1):42-8.

6. Moon JS, Lee SY, Kim JH, Choi YH, et al. Synergistic alveolar bone resorption by diabetic advanced glycation end products and mechanical forces. J Periodontol. 2019 Dec;90(12):1457-1469.

7. Andrés-Veiga M, Barona-Dorado C, Martínez-González MJ, et al. Influence of the patients' sex, type of dental prosthesis and antagonist

\section{CONCLUSIONS}

The retrospective study aimed to propose a method of analysis of real resorptions from daily practice, using current radiographs in optimal information / exposure ratio.

The method was based on the size of the implant and the common element in two radiographs and can be generalized to other studies.

In the cases analysed, the various techniques applied were not differentiated, and we are interested in an overview of the results that validate the working protocol.

Similar results measured in the two groups also demonstrate that alveolar remodelling does not depend on the type of implant and that the density of newly formed peri-implant bone tends to equal the density of the area.

The results obtained from the measurements show that half of the post-extraction sites did not have any vertical resorption of the alveolar process; another quarter showed barely perceptible clinical resorption; In the last quarter were registered all the notable resorptions that were associated with various factors (temporary removable prosthesis, surgical flap, medication and small health problems, etc.) showing real situations, not ideal conditions.

It seems that there is no statistical correlation between vertical resorption and alveolo-implant space (gap).

Extensive studies can verify the correlation of resorption with implant diameter or other parameters.

\section{Note}

The authors have equal contributions to the development of this article.

Conflict of interest: none declared Financial support: none declared

on residual bone resorption at the level of the premaxilla. Med Oral Patol Oral Cir Bucal. 2012 Jan 1;17(1):e178-82.

8. Chen $\mathrm{H}$, Zhang $\mathrm{G}$, WeigI P, Gu X. Immediate placement of dental implants into infected versus noninfected sites in the aesthetic zone: $A$ systematic review and meta-analysis. J Prosthet Dent. 2018 Nov;120(5):658-667.

9. Naji BM, Abdelsameaa SS, Alqutaibi AY, Said Ahmed WM. Immediate dental implant placement with a horizontal gap more than two millimeters: a randomized clinical trial. Int J Oral Maxillofac Surg. 2021 May;50(5):683-690.

10. Ahmed KE. Monitoring Alveolar Ridge Remodeling Post-Extraction Using Sequential Intraoral Scanning over a Period of Four Months. Int J Environ Res Public Health. 2020 Sep 11;17(18):6638.

11. Li Y, Huang B, Cao J, Fang T, Liu G, Li X, Wu J. Estimating radiation dose to major organs in dental X-ray examinations: A phantom study. Radiat Prot Dosimetry. 2020 Dec 30;192(3):328-334.

12. Lee SM, Lee SS, Huh KH, Yi WJ, Heo MS, Choi SC. The effects of location of alveolar crest on the vertical bone heights on panoramic radiographs. Dentomaxillofac Radiol. 2012 Feb;41(2):117-21. 